\title{
Immunoinflammatory markers and disease activity in systemic lupus erythematosus: something old, something new
}

M.A. Elwy, Z.A. Galal'² and H.E. Hasan ${ }^{3}$

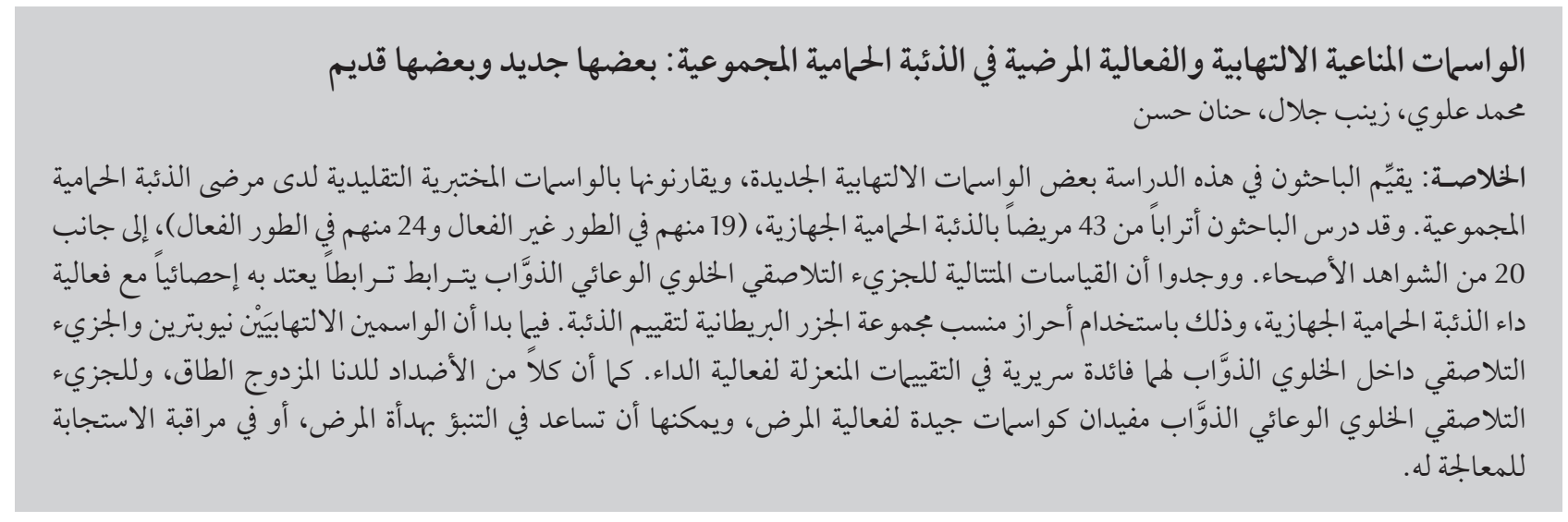

ABSTRACT This study assessed the utility of some novel inflammatory markers compared with traditional laboratory markers in patients with systemic lupus erythematosus (SLE). In a cohort of 43 SLE patients (19 with inactive and 24 with active SLE) and 20 healthy controls, serial measures of soluble vascular cell adhesion molecule (sVCAM-1) were significantly associated with SLE disease activity, scored using the British Isles Lupus Assessment Group index. Inflammatory markers neopterin and soluble intercellular adhesion molecule (sICAM-1) appeared to be clinically useful for isolated assessments of disease activity. Both antibodies to double-stranded DNA (antidsDNA) and sVCAM-1 were relatively good markers of disease activity and could help to predict remission or monitor the therapeutic response in SLE.

Marqueurs immuno-inflammatoires - anciens et nouveaux - et évolutivité du lupus érythémateux disséminé RÉSUMÉ La présente étude a évalué l'utilité de nouveaux marqueurs immuno-inflammatoires par rapport aux marqueurs de laboratoire conventionnels chez des patients atteints de lupus érythémateux disséminé (LED). Dans une cohorte de 43 patients atteints de lupus érythémateux disséminé (19 présentant un lupus érythémateux disséminé stable et 24 un lupus érythémateux disséminé évolutif) et un groupe témoin comprenant 20 sujets sains, la présence répétée de molécules d'adhésion des cellules vasculaires sous forme soluble (sVCAM-1) était associée de manière significative à l'activité du lupus érythémateux disséminé. Ces mesures ont été effectuées selon l'indice du British Isles Lupus Assessment Group (Groupe d'évaluation du lupus des îles britanniques). Les marqueurs inflammatoires comme la néoptérine et la molécule d'adhésion intercellulaire (sICAM-1) se sont révélés utiles sur le plan clinique pour réaliser des évaluations isolées de l'activité de la maladie. Les anticorps anti-ADN double brin (anti-dsDNA) ainsi que les sVCAM-1 se sont avérés être de bons marqueurs d'activité de la maladie contribuant à prévoir une rémission ou à surveiller la réponse thérapeutique du lupus érythémateux disséminé.

${ }^{7}$ Department of Rheumatology and Rehabilitation; ${ }^{2}$ Department of Clinical Pathology; ${ }^{3}$ Department of Internal Medicine, Faculty of Medicine, Ain Shams University, Cairo, Egypt (Correspondence to M.A. Elwy: elwy.mohamed@yahoo.com).

Received: 27/03/08; accepted: 06/05/08 


\section{Introduction}

Systemic lupus erythematosus (SLE) is a progressive autoimmune disorder associated with chronic stimulation of various components of the immune system, affecting the skin, joints, kidneys, heart and nervous and haematopoietic systems. The etiology of SLE is unknown and its clinical course is highly variable, with periods of flare-ups and remission $[1,2]$. A characteristic feature of SLE is that the pattern of organ involvement differs from one patient to another, suggesting differences in pathogenic mechanisms, a hypothesis supported by differences in the autoantibody profiles that correlate with involvement of different organs [2-4]. The clinical evaluation of the disease still lacks a reliable marker that is both sensitive and specific for the diagnosis as well as for the measurement of disease activity $[5,6]$.

The accepted measures of disease activity in SLE include erythrocyte sedimentation rate (ESR), plasma/serum complement component $3(\mathrm{C} 3)$ and component 4 (C4) and presence of antibodies to double-stranded DNA (anti-dsDNA). Some patients, however, have abnormalities in these tests for considerable periods yet show few clinical symptoms or functional deterioration of a major organ; others are markedly symptomatic with only minor aberrations in these test results $[4,7,8]$.

The utility of some relatively new markers of SLE disease activity are still disputed [2]. Neopterin is specifically produced by human macrophages when stimulated by interferon-gamma released from activated T-lymphocytes, and is therefore an indirect marker of the upregulation of the cellular immune system $[9,10]$. Intracellular adhesion molecule-1 (ICAM-1) and vascular cell adhesion molecule-1 (VCAM-1) are members of the immunoglobulin supergene family and play a central role in cell-to-cell and in cell-to-extracellular matrix-mediated immune responses [11]. During exacerbations of SLE, endothelial cells are activated to increase their expression of adhesion molecules $[12,13]$.

The objectives of this study were to compare some of the newer markers (neopterin, sICAM-1, sVCAM-1) with conventionallaboratory measures (antidsDNA, C3 and C4) and the nonspecific inflammatory marker C-reactive protein (CRP), using a standard index of disease activity, and to investigate whether certain variables correlated with any type of specific organ involvement in an unselected group of patients with SLE.

\section{Methods}

\section{Sample}

The study group were patients who were regular attendees at the outpatient clinics of the rheumatology units of the department of internal medicine and the department of rheumatology and rehabilitation at Ain Shams University Hospitals, Cairo, Egypt between August 2005 and March 2007. A convenience sample of 43 patients ( 40 females and 3 males) who were present at the time of the researchers' visit and who agreed to participant were selected. All of them fulfilled 4 or more of the 1982 revised American Rheumatism Association (ARA) criteria for the classification of SLE [14]. Their mean age was 41.0 [standard deviation (SD)] 13.2 years and the mean disease duration of the patients at the time of recruitment was 10.7 (SD 9.4) years. A control group of 20 age- and sex-matched, apparently healthy individuals ( 18 females and 2 males) was also selected for the study; their mean age was 38.1 (SD 9.3) years. All participants were informed and willing to be included in the study.

\section{Data collection}

\section{Clinical}

All the patients had documented medical histories of SLE and were subjected to a full history-taking and thorough clinical examination for this study by qualified internists. Case documentation, assessment of disease activity, damage events and therapeutic interventions were made by the same group of rheumatologists throughout.

In this study the overall disease activity was scored using the British Isles Lupus Assessment Group (BILAG) index of disease activity [15] to assess lupus activityin the major organ systems (mucocutaneous, musculoskeletal, renal, nervous, cardiovascular, vasculitis and haematological) and general constitutional manifestations. All the organ systems were graded $A$ to $E$ and then to obtain a global BILAG score the component scores were assigned numerical values $[$ A (most active disease $)=9, \mathrm{~B}$ (intermediate activity) $=3, \mathrm{C}$ (mild and stable disease activity) $=1, \mathrm{D}$ (inactive disease $)=0$ and $\mathrm{E}$ (no activity ever $)=$ $0]$, resulting in a potential global score ranging from 0 to 72 . This numerical score as well as the total BILAG score have been shown to be valid $[10,16]$. SLEpatients were arbitrarily categorized into 2 groups: active disease (BILAG score $>5$ ) and inactive disease (BILAG $\leq 5$ ). A total BILAG score $>5$ usually consists of at least 1 organ score of 3 (which signifies 1 organ system with a minor flare-up).

Renal lesions were classified histologically according to the World Health Organization (WHO) classification for lupus glomerulonephritis [17]. Hypertension was also diagnosed according to WHO definitions. Systolic and diastolic blood pressure (BP) were determined using an average of 2 consecutive sitting BP readings taken 5 minutes apart. Patients were considered to be hypertensive if over the study period they had mean systolic BP > $140 \mathrm{mmHg}$ and/or diastolic $\mathrm{BP}>90 \mathrm{mmHg}$ and/or were taking antihypertensive drugs.

None of the patients or controls had a concomitant viral or bacterial infection or other disorders such as diabetes mellitus, liver or thyroid disease at the time of the study. Subsequent follow-up supported this clinical opinion. None 
of the participants was pregnant or had drug-induced lupus erythematosus at the time of the study.

\section{Laboratory methods}

The following laboratory investigations were completed for all the participants:

- ESR and complete blood count (CBC) using standard methods.

- Serum creatinine, blood urea, liver function tests, fasting lipid profiles and fasting blood sugar were determined using an autoanalyser (Synchron CX-7, Beckman).

- Complete urine analysis for the presence of red blood cells (RBCs), pus cells, albumin and urinary casts (either granular, hyaline, tubular and/or mixed type).

- CRP detected by ELISA technique (Active Diagnostic Systems), with normal levels defined as $<6 \mathrm{mg} / \mathrm{L}$.

- Antinuclear antibodies (ANA) and anti-dsDNA were detected by indirect immunofluorescent assay on mouse kidney and stomach slides and Crithidia luciliae slides (Immco Diagnostics). The slides were analysed with a Nikon epifluorescent microscope. A titre $\geq 1: 80$ was considered positive for ANA and $>1: 40 \mathrm{IU} / \mathrm{mL}$ for anti-dsDNA detection.

- Complement C3 and C4 were determined by single radial immunodiffusion plates (Diffu-Plate, Biocientifica).

- Soluble VCAM-1 and ICAM-1 were quantified with an immunoassay kit (R \& D Systems), with normal levels for sICAM-1 defined as 115-306ng/ $\mathrm{mL}$ [mean 211 (SD 2SD) ng/mL] and for sVCAM-1 as 349-991 ng/ $\mathrm{mL}$ [mean 557 (SD 139.6) ng/mL].

- Detection of serum neopterin was performed using an enzyme immunoassay kit (DRG Instruments), following the basic principles of competitive ELISA. The serum neopterin normal value was $<10 \mathrm{nmol} / \mathrm{L}(<2.5$ $\mathrm{ng} / \mathrm{mL}$ ), with the range of normal values $3-9 \mathrm{nmol} / \mathrm{mL}$.
Blood and urine samples from SLE patients and controls were obtained at the time of recruitment to the study. All blood specimens were collected under aseptic conditions and were allowed to clot for about $30 \mathrm{~min}$ at room temperature. Sera were obtained after centrifugation, and routine laboratory tests were carried out immediately. The remaining sera were stored frozen in aliquots at $-20{ }^{\circ} \mathrm{C}$ for the subsequent assays and thawed only once. Grossly haemolytic, icteric or grossly lipaemic specimens were discarded to avoid aberrant results; repeated freeze-thaw cycles were avoided. Stored samples were protected from light. Urinary sediment analysis was carried out by routine microscopy.

The samples of the SLE cohort and controls were analysed together at the same time to avoid methodological errors.

All markers and the disease activity index were measured at 3 time points over 6 months, at the beginning (the baseline measurement) and 3 and 6 months after joining the study.

\section{Data analysis}

Statistical analysis of the data was performed with SPSS, version 11.0 software, and continuous variables were presented as means and SD. Comparisons between the SLE disease groups and the control group were carried out using the Mann-Whitney test. The relationship between inflammatory markers levels and clinical parameters was determined using the Spearman correlation analysis and the linear regression method. $P$ values $<0.05$ were considered to be statistically significant.

\section{Results}

\section{Background characteristics of patients}

Using the BILAG score cutoff $>5$ to separate active from inactive SLE, 24 (55.8\%) patients were classified with active disease at the time of investigation and $19(44.2 \%)$ with inactive disease.
Of the 24 patients with active disease, 16 patients had active extrarenal disease and 8 had active renal disease defined by the presence of: persistent proteinuria $>0.5 \mathrm{~g} /$ day or dipstick proteinuria $>3+$ if measurement was not performed; or cellular urinary casts (haeme, granular, red cell or mixed) and cells present (> 5 RBCs or WBCs per high-power field); or unexplained rise in serum creatinine $(>1.5 \mathrm{mg} / \mathrm{dL})$. Of these 8 patients, 5 patients had a renal biopsy: 3 were diagnosed with diffuse proliferative nephritis (class IV) and 2 with focal segmental nephritis (class III). Of the remaining 3 patients with active renal disease, 1 had thrombocytopenia that was not considered safe for the biopsy procedure and 2 had lupus nephritis with clear clinical and laboratory findings.

A total of 39 (90.7\%) patients were receiving drugs at the time of assessment: 13 were on low-dose corticosteroids (prednisone $47.5 \mathrm{mg} /$ day), 4 on antimalarials, 3 on methotrexate and 1 on azathioprine as the single treatment. The remaining 18 patients were receiving combined drug treatments: 2 were on antimalarials plus low-dose corticosteroid, 1 on methotrexate plus corticosteroid (> $7.5 \mathrm{mg} /$ day), 6 on azathioprine plus corticosteroid ( $>7.5$ $\mathrm{mg} /$ day), 5 on cyclophosphamide plus corticosteroid (>7.5 mg/day) and 4 on cyclosporin A plus corticosteroid (> 7.5 $\mathrm{mg} /$ day).

All the 5 patients treated with cyclophosphamidehadactivelupus nephritis: 2 were classified as IV.B and 1 was III.B according to the WHO classification. A renal biopsy was not performed on $2 \mathrm{pa}-$ tients, but their kidney involvement was obvious from the clinical and laboratory findings (proteinuria $>1 \mathrm{~g} /$ day). Of the 4 patients treated with cyclosporin A, lupus nephritis was present in 3 , and was documented by histology in 2 cases (1 III.A and 1 IV.B); in 1 of them the presence of lupus nephritis was clear from the clinical and laboratory findings. There were 3 patients treated with 
cyclophosphamide and 1 treated with cyclosporine A, who received immunosuppressive therapy before inclusion in the study.

\section{Organ system involvement}

Of the 24 patients with active disease, 15 (62.5\%) had involvement of only 1 organ system and $9(37.5 \%)$ had involvement of more than 1 organ system. Large variations in disease activity were found. On average, $38 \%$ of the patients showed no activity ever in at least 1 of the organ systems (category E). The active and inactive SLE groups did not differ significantly in terms of age, disease duration and number of ARA criteria fulfilled (Student $t$-test) (Table 1). In this study more patients had activity (categories A to C) in the musculoskeletal, mucocutaneous and haematological systems (22,21 and 19 respectively) as compared with the renal, neurological and cardiovascular systems (10,4 and 5 respectively) (Table 2).

\section{Laboratory markers}

Table 3 shows the data for the conventional laboratory measures of inflammation and SLE disease activity (elevated CRP level, positive antidsDNA titre and C3 and C4 levels) compared with the novel immunoinflammatory markers. As expected, the proportion of patients with elevated CRP level was significantly different

$\begin{aligned} & \text { Table 1 Characteristics of patients with active and inactive systemic lupus } \\
& \text { erythematosus (SLE) }\end{aligned}$
\begin{tabular}{|lcc|}
\hline Variable & $\begin{array}{c}\text { Active SLE } \\
(\boldsymbol{n}=\mathbf{2 4})\end{array}$ & $\begin{array}{c}\text { Inactive SLE } \\
(\boldsymbol{n}=\mathbf{1 9})\end{array}$ \\
Mean (SD) age (years) & $42.3(14.7)$ & $40.1(11.9)$ \\
Mean (SD) disease duration (years) & $11.1(7.1)$ & $10.4(9.1)$ \\
No. of ARA criteria & 6.4 & 5.2 \\
\hline
\end{tabular}

$S D=$ standard deviation $; A R A=$ American Rheumatism Association.

between SLE patients and the control group and between inactive and active SLE patients $(P<0.001)$. The percentage of anti-dsDNA-positive patients was not significantly different between the 2 SLE groups. None of the controls had anti-dsDNA antibodies in their sera. No significant differences were found in the mean serum levels of the complement $\mathrm{C} 3$ and $\mathrm{C} 4$ in those with inactive versus active SLE.

The mean levels of neopterin, sICAM-1 and sVCAM-1 were higher in SLE patients compared with controls $(P<0.01)$. Mean levels of neopterin and sVCAM-1 were significantly different comparing active and inactive SLE groups $(P<0.05)$.

\section{Correlations between different laboratory markers}

Significant positive correlations between the different markers are shown on Table 4. There were significant correlations between neopterin and CRP levels $(P<0.05)$, between neopterin and dsDNA antibody level $(P<0.001)$ and between neopterin and SVCAM-1 levels $(P<0.001)$.

In addition, correlations were calculated between the different serological markers and the total BILAG score. There was a significant correlation between total BILAG score and CRP level $(P<0.001)$, but not between total BILAG score and anti-dsDNA or C3 and $\mathrm{C} 4$ levels. Also, no correlation was found between total BILAG score and sICAM-1 levels, but significant correlations were found with SVCAM-1 $(P<0.01)$ and neopterin $(P<0.05)$ levels (Table 4).

To calculate the correlations between the serological markers and disease activity of different BILAG organ systems, we chose the 3 organ systems where the number of patients with a BILAG score $>3$ was high enough $(n$ >9) for a statistical analysis (i.e. mucocutaneous, musculoskeletal and renal involvement). No positive correlation was found between any of the tested

\begin{tabular}{|c|c|c|c|c|c|}
\hline \multirow[t]{2}{*}{ Organ system } & \multicolumn{5}{|c|}{ SLE disease activity (no. of patients) } \\
\hline & $\begin{array}{c}\text { A } \\
\text { Strong }\end{array}$ & $\begin{array}{c}\text { B } \\
\text { Moderate }\end{array}$ & $\begin{array}{c}\text { C } \\
\text { Low }\end{array}$ & $\begin{array}{c}\text { D } \\
\text { None } \\
\text { presently }\end{array}$ & $\begin{array}{c}\text { E } \\
\text { None ever }\end{array}$ \\
\hline Musculoskeletal & 1 & 4 & 17 & 15 & 6 \\
\hline Mucocutaneous & 1 & 9 & 11 & 14 & 8 \\
\hline Haematological & 0 & 3 & 16 & 14 & 10 \\
\hline Vasculitis & 0 & 0 & 13 & 12 & 18 \\
\hline Renal & 1 & 6 & 3 & 13 & 20 \\
\hline General & 1 & 0 & 4 & 23 & 15 \\
\hline Nervous system & 0 & 0 & 4 & 8 & 31 \\
\hline Cardiovascular/ respiratory & 0 & 0 & 5 & 15 & 23 \\
\hline
\end{tabular}




\begin{tabular}{|c|c|c|c|c|}
\hline Marker & $\begin{array}{c}\text { Controls } \\
(n=20)\end{array}$ & $\begin{array}{l}\text { Active SLE }^{a} \\
(n=24)\end{array}$ & $\begin{array}{l}\text { Inactive SLE } \\
\quad(n=19)\end{array}$ & $P$-value \\
\hline Elevated CRP level [no. (\%) of patients] & $1(5.0)$ & $17(70.8)$ & $5(26.3)$ & $<0.05^{b, c}$ \\
\hline Positive anti-dsDNA [no. (\%) of patients] & $0(0.0)$ & $15(62.5)$ & $9(47.3)$ & $<0.05^{\mathrm{b}}$ \\
\hline Mean C3 level (g/L) & 1.23 & 0.64 & 0.89 & $<0.05^{\mathrm{b}}$ \\
\hline Mean C4 level (g/L) & 0.31 & 0.16 & 0.15 & $<0.05^{\mathrm{b}}$ \\
\hline Mean neopterin level (nmol/L) & 6.7 & 14.3 & 10.1 & $<0.05^{\mathrm{b}, \mathrm{c}}$ \\
\hline Mean sICAM-1 level (ng/mL) & 275.1 & 669.7 & 490.6 & $<0.05^{\mathrm{b}}$ \\
\hline Mean sVCAM-1 level (ng/mL) & 782 & 1721 & 1465 & $<0.05^{\mathrm{b}, \mathrm{c}}$ \\
\hline
\end{tabular}

${ }^{a}$ Total BILAG score $>5$.

${ }^{b} P<0.05$ between SLE patients and controls; ${ }^{c} P<0.05$ between active and inactive SLE patients.

$C R P=C$-reactive protein; anti-dsDNA = antibodies to double-stranded DNA; $C 3$ and $C 4=$ complement components 3 and $4 ;$ s $C$ CAM-1 = soluble intercellular adhesion molecule; sVCAM-1 = soluble vascular cell adhesion molecule.

markers and the disease activity of different BILAG organ systems. Higher levels of neopterin and sVCAM-1 were identified in patients with lupus nephritis (mean $14.8 \mathrm{nmol} / \mathrm{L}$ and 1780 $\mathrm{nmol} / \mathrm{L}$ respectively) compared with patients without kidney impairment (mean $13.7(\mathrm{ng} / \mathrm{mL})$ and $1710(\mathrm{ng} /$ $\mathrm{mL}$ ) respectively).

\section{Serial measurements of markers and disease activity index}

Among the 43 SLE patients complete follow-up data were only available for 22 patients who were included in the next step, in which all markers and disease activity index were measured at 3 time points over 6 months, at the beginning (the baseline measurement) and after 3 and 6 months. They were divided into 3 subgroups (active with flare-ups, active with remission and inactive patients). Patients with lupus nephritis were present in both active subgroups with initially high disease activity. However, they were not homogeneous enough to be included in the final evaluation as a separate subgroup. The descriptive statistics for anti-dsDNA, complement $\mathrm{C} 3$ and $\mathrm{C} 4$, neopterin, sICAM- 1 and SVCAM- 1 levels in the subgroups over the 6 months are shown in Table 5.

The serum levels of anti-dsDNA antibodies were the lowest in the inactive subgroup. A significant difference in anti-dsDNA levels was found between the inactive subgroup and the subgroup with active flare-ups at baseline, 3 months and 6 months ( $P$ $<0.001)$. The inactive subgroup and the active with remission subgroup differed significantly in the baseline measurements $(P<0.001)$. Among the subgroup who were active with remission a significant difference was found between measures at baseline and 6 months $(P>0.01)$.

The serum levels of $\mathrm{C} 3$ were the highest in the inactive subgroup, but differences between the subgroups did not reach significant levels, nor were there significant differences within the subgroups over the 3 measurements. The highest levels of $\mathrm{C} 4$ were in the inactive subgroup, but the differences were not statistically significant. Changes in C4 levels at follow-up within each subgroup were not significant.

The differences in serum neopterin levels were significant comparing the inactive subgroup and both active subgroups at baseline and at 6 months ( $P$ $<0.01$ and $P<0.001$ respectively). There were no significant differences between the active subgroups when compared with each other or when the changes within each subgroup were compared.

The highest levels of sVCAM-1 were in the subgroup with active flareups at baseline. Although the differences between active subgroups were not significant statistically, significant decreases between baseline and the 6-month measurements of SVCAM-1 levels were

\begin{tabular}{|c|c|c|c|c|c|c|}
\hline \multirow[t]{2}{*}{ Marker } & \multicolumn{6}{|c|}{ Marker } \\
\hline & CRP & anti-dsDNA & Neopterin & sICAM-1 & sVCAM-1 & C3 \\
\hline CRP & 1 & - & - & - & - & - \\
\hline anti-dsDNA & - & 1 & - & - & - & - \\
\hline Neopterin & $<0.05$ & $<0.001$ & 1 & - & - & - \\
\hline sICAM-1 & - & - & - & 1 & - & - \\
\hline sVCAM-1 & - & - & $<0.001$ & - & 1 & - \\
\hline C3 & - & - & - & - & - & 1 \\
\hline BILAG score & $<0.001$ & - & $<0.05$ & - & $<0.01$ & - \\
\hline
\end{tabular}

$C R P=C$-reactive protein; anti-dsDNA = antibodies to double-stranded $D N A ; s / C A M-1=$ soluble intercellular adhesion molecule; sVCAM-1 = soluble vascular cell adhesion molecule; C3=complement component 3;

$B I L A G=$ British Isles Lupus Assessment Group index . 


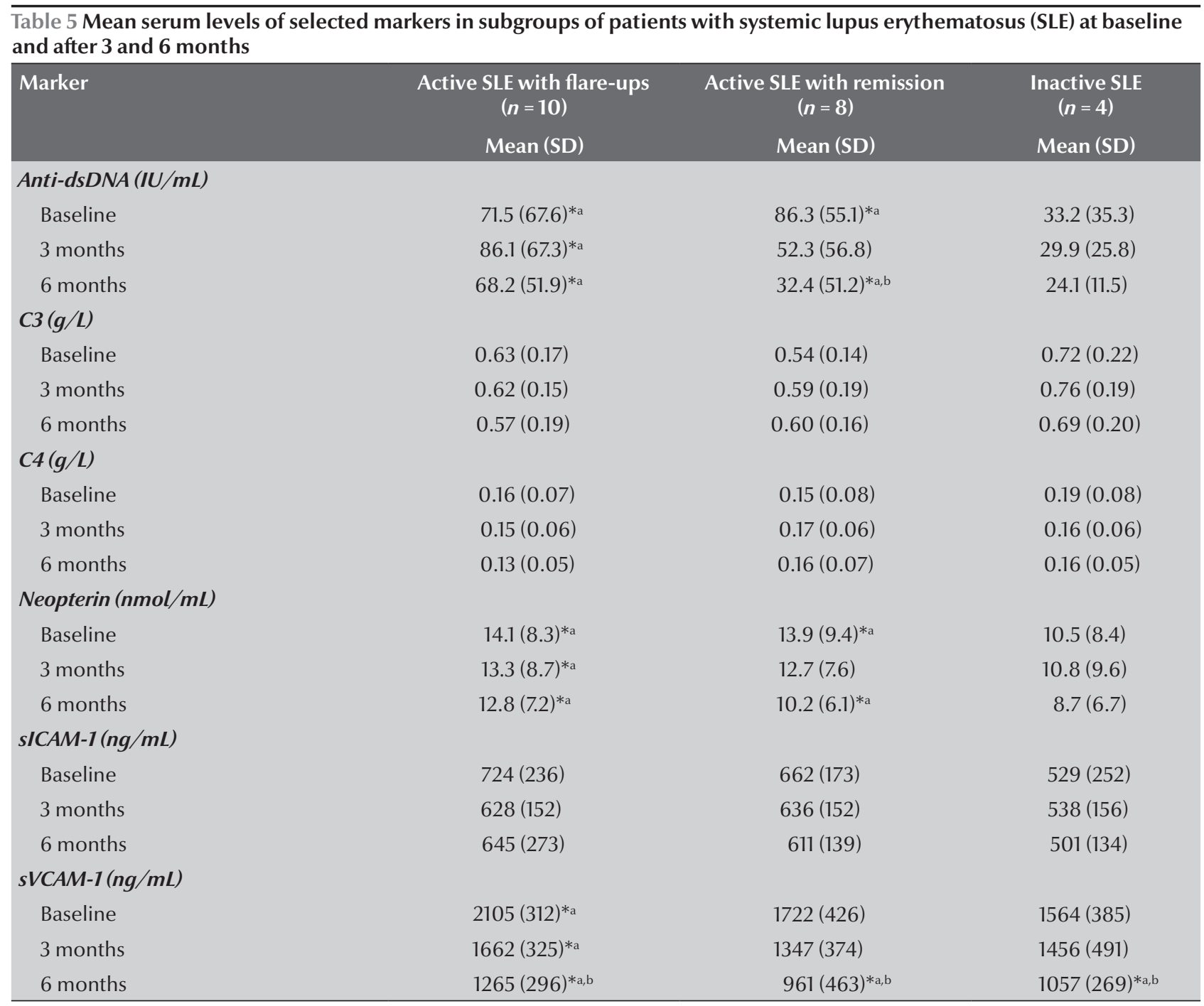

${ }^{* a} P<0.05$ versus inactive subgroup; ${ }^{* b} P<0.05$ versus baseline measurement.

$S D=$ standard deviation; anti-dsDNA = antibodies to double-stranded DNA; C3 and C4 = complement components 3 and 4; s/CAM-1 = soluble intercellular adhesion molecule; sVCAM-1 = soluble vascular cell adhesion molecule.

found within all subgroups $(P<0.001, P$ $<0.001$ and $P<0.01$ respectively).

No significant differences in sICAM-1 levels were found among subgroups or within subgroups.

\section{Discussion}

Levels of complement $\mathrm{C} 3$ and $\mathrm{C} 4$, and dsDNA antibodies, classic laboratory markers for disease activity in SLE, did not differentiate between active and inactive disease in our patient cohort. This was in agreement with other studies suggesting that serum neopterin $[2,18,19]$ and sVCAM- $1[2,13,20,21]$ are more sensitive markers of disease activity.

Levels of anti-dsDNA antibodies are a widely accepted measure for evaluating disease activity in SLE. However, anti-dsDNA does not necessarily fluctuate with disease activity and a substantial proportion of SLE patients are anti-dsDNA negative [4]. SLE is characterized by hyperactivity of $\mathrm{B}$ cells and by the production of organ non-specific autoantibodies. Levels of anti-dsDNA antibodies are very specific tests, particularly in diagnosis $[7,8]$. In this study, the correlation of anti-dsDNA with the disease activity index was weaker than in the case of SVCAM-1. In some studies the limited value of serial measurement of anti-dsDNA antibodies for the prediction of lupus relapse has been discussed $[2,13]$.

The complement components C3 and $\mathrm{C} 4$ are also traditional markers of disease activity and their use reflects the important role that the complement system plays in SLE pathogenesis $[5,6]$. An impaired complement system might affect the clearance of apoptotic material and thus enhance the vicious circle in SLE, but the clinical measurement of complement components is not sensitive enough to detect disease activity in SLE $[2,18]$. According to the results of our study, their levels did not correlate significantly with the disease activity index. $\mathrm{C} 3$ and $\mathrm{C} 4$ did 
not decrease significantly during SLE disease flare-ups in individual patients, even though their levels correlated (but not significantly) with the BILAG score. Furthermore, their levels did not change significantly and rapidly enough with the declining activity over the period of the follow-up in the subgroup with declining BILAG index. Their utility as a reliable marker of disease activity and therapy response is therefore limited [18].

In accordance with some earlier studies, sICAM-1 levels were elevated above the normal range in all our patients but did not differ significantly between patients with active and inactive disease $[2,6,18,22]$. It is noteworthy that neither the values of the $\mathrm{C} 3$ and C4 nor the levels sICAM-1 correlated significantly with the disease activity index and this agrees with other studies $[16,22]$. These results contrast with data reported in other studies [16,23].

Of all the immunoinflammatory variables we investigated, CRP, neopterin and SVCAM- 1 correlated best with disease activity measured by the total BILAG score. Interestingly, several studies of CRP autoantibodies have claimed that serum levels of CRP often remain low despite high disease activity and despite high levels of other acute phase proteins [24], suggesting that the presence of low CRP levels may not reflect the presence of antibodies to CRP which may play an important role in SLE pathogenesis [25].

The important question is whether the involvement of different organ systems in SLE correlates with different patterns of immunoinflammatory markers. This is likely, as it is well known that anti-dsDNA correlates with renal disease, but not with musculoskeletal involvement in SLE [6].

We chose the BILAG scoring scale because it gives an accurate organspecific measurement of disease activity, and would allow correlation of immunoinflammatory markers with disease activity in the different organs involved. However, we could not establish a relationship between any of the tested markers and organ-specific BILAG scores. This may have been because of the limited numbers of patients in each group and the wide variety of organ-specific BILAG groups included in our study (7 groups).

Our results contrast with other studies where, for example, changes in anti-dsDNA and complement concentrations were reported predominately to accompany flare-ups of lupus nephritis $[20,26,27]$. Significant correlations have been found between other biological laboratory markers and some BILAG organ system scores, such as between interleukin-1 receptor antagonist (IL1ra), and the musculoskeletal score [7] and between soluble tumour necrosis factor receptors p55 and p75 (sTNFR55/sTNFR-75) and renal BILAG score [6]. Their findings, but not ours, support the hypothesis of different pathological mechanisms in the different organ systems.

Although we found higher values of sVCAM-1 in patients with lupus nephritis compared with patients without kidney impairment the differences were not statistically significant, a result that agrees with other studies [27-29]. However, another study showed that the serum level of sVCAM-1 was significantly higher in patients with active lupus nephritis (WHO classes III and IV) than in patients in inactive lupus nephritis and there was a positive correlation between SVCAM-1 and SLE disease activity, which decreased during remission [20]. Similarly, an increased level of neopterin was reported with lupus nephritis [10].

In this study, time-series modelling was adopted to investigate if serial measurements of some inflammatory markers could predict the BILAG score and hence SLE disease activity in individual patients. Time-series analysis showed that sVICAM-1 increased significantly during flare-ups in SLE disease activity. Similarly, a significant decrease in concentrations was observed in patients with active SLE who went into disease remission, as shown in another study [20]. In those patients whose disease remained active or in remission on the third successive assessment, concentrations were found to be correspondingly greater or smaller compared with initial values.

Levels of sICAM-1 were predominantly within the baseline range during maximal disease activity and did not change in the time period before remission. These findings might in part be explained by binding of the functionally active soluble molecules to their respective ligands on activated leukocytes [13]. The present finding of high sVCAM-l levels, however, conflicts with this hypothesis, although it might be explained by an excess of released molecules compared with the number of accessible molecules $[13,23,29,30]$. Generally, none of the other study parameters was found to mirror SLE disease activity as effectively as SVCAM-1 using both single time-point and timeseries analyses.

In conclusion, this study indicates that serial concentrations of sVCAM- 1 are significantly associated with SLE disease activity scored using the BILAG index. Measurement of neopterin and sVCAM-1 appeared to be clinically useful for isolated assessments of disease activity. Both anti-dsDNA and sVCAM-1 were relatively good markers of disease activity in SLE. It seems that both are suitable for the monitoring of disease activity and could help to predict remission or to monitor the therapeutic response. Assessment of the possible advantages of sVCAM- 1 over anti-dsDNA requires additional information from future studies. 


\section{References}

1. De Leeuw $\mathrm{K}$ et al. Traditional and non-traditional risk factors contribute to the development of accelerated atherosclerosis in patients with systemic lupus erythematosus. Lupus, 2006, 15(10):675-682.

2. Horak $\mathrm{P}$ et al. Clinical utility of selected disease activity markers in patients with systemic lupus erythematosus. Clinical Rheumatology, 2001, 20(5):337-434.

3. Kaplanski $\mathrm{G}$ et al. Increased soluble vascular cell adhesion molecule 1 concentrations in patients with primary or systemic lupus erythematosus-related antiphospholipid syndrome: correlations with the severity of thrombosis. Arthritis and Rheumatism, 2000, 43(1):55-64.

4. Rahman A, Hiepe F. Anti-DNA antibodies--overview of assays and clinical correlations. Lupus, 2002, 11(12):770-773.

5. Kyttaris VC, Juang YT, Tsokos GC. Immune cells and cytokines in systemic lupus erythematosus: an update. Current Opinion in Rheumatology, 2005, 17(5):511-512.

6. Wais $\mathrm{T}$ et al. Subclinical disease activity in systemic lupus erythematosus: immunoinflammatory markers do not normalize in clinical remission. Journal of Rheumatology, 2003, 30(10):2133-2139.

7. Lim KL et al. Urine neopterin as a parameter of disease activity in patients with systemic lupus erythematosus: comparisons with serum sIL-2R and antibodies to dsDNA, erythrocyte sedimentation rate, and plasma C3, C4, and C3 degradation products. Annals of the Rheumatic Diseases, 1993, 52(6):429-435.

8. Nagy G et al. Diagnostic value of combined evaluation of neopterin and anti-DNA antibody levels for assessment of disease activity in systemic lupus erythematosus. Clinical and Experimental Rheumatology, 2000, 18(6):699-705.

9. Jin $\mathrm{O}$ et al. Lymphocyte apoptosis and macrophage function correlation with disease activity in systemic lupus erythematosus. Clinical Rheumatology, 2005, 24(2):107-110.

10. Lhee HY et al. The clinical significance of serum and urinary neopterin levels in several renal diseases. Journal of Korean Medical Science, 2006, 21:678-682.

11. Tso TK, Huang WN. Elevated soluble intercellular adhesion molecule-1 levels in patients with systemic lupus erythematosus: relation to insulin resistance. Journal of Rheumatology, 2007, 34(4):726-730.

12. Belmont HM et al. Up-regulation of endothelial cell adhesion molecules characterizes disease activity in systemic lupus erythematosus. Arthritis and Rheumatism, 1994, 37(3):376-383

13. Spronk PE et al. Levels of soluble VCAM-1, soluble ICAM-1, and soluble E-selectin during disease exacerbations in patients with systemic lupus erythematosus (SLE), a long term prospective study. Clinical and Experimental Immunology, 1994, 97(3):439-444.

14. Tan EM et al. The 1982 revised criteria for the classification of systemic lupus erythematosus. Arthritis and Rheumatism, 1982, 25:1271-1277.

15. May EM et al. The BILAG index a reliable and valid instrument for measuring clinical disease activity in systemic lupus erythematosus. Quarterly Journal of Medicine, 1993, 86:447-458.
16. Stoll T et al. Further validation of the BILAG disease activity index in patients with systemic lupus erythematosus. Annals of the Rheumatic Diseases, 1996, 55:756-760.

17. Pirani CL, Pollock VT. Systemic lupus erythematosus glomerulonephritis (lupus nephritis). In: Cluskey RT, Andres GA, eds. Immunologically Mediated Renal Disease. New York, Marcel Dekker, 1978.

18. Mahmoud RA, El-Gendi HI, Ahmed HH. Serum neopterin, tumor necrosis factor-alpha and soluble tumor necrosis factor receptor II (p75) levels and disease activity in Egyptian female patients with systemic lupus erythematosus. Clinical Biochemistry, 2005, 38(2):134-141.

19. Samsonov MY et al. Serum soluble markers of immune activation and disease activity in systemic lupus erythematosus. Lupus, 1995, 4(1):29-32.

20. Ikeda $Y$ et al. Relationship between lupus nephritis activity and the serum level of soluble VCAM-1. Lupus, 1998, 7(5):347-354.

21. Zaccagni $\mathrm{H}$ et al. Soluble adhesion molecule levels, neuropsychiatric lupus and lupus-related damage. Frontiers in Bioscience, 2004, 9:1654-1659.

22. Wellicome $\mathrm{SM}$ et al. Detection of a circulating form of vascular cell adhesion molecule-1: raised levels in rheumatoid arthritis and systemic lupus erythematosus. Clinical and Experimental Immunology, 1993, 92(3):412-418.

23. Sari RA et al. Correlation of serum levels of soluble intercellular adhesion molecule-1 with disease activity in systemic lupus erythematosus. Rheumatology International, 2002, 21(4):149-152.

24. Sjowall C, Wettero J. Pathogenic implications for autoantibodies against C-reactive protein and other acute phase proteins. Clinica Chimica Acta, 2007, 378(1-2):13-23.

25. Rosenau BJ, Schur PH. Antibodies to C reactive protein. Annals of the Rheumatic Diseases, 2006, 65(5):674-676.

26. Molad $\mathrm{Y}$ et al. Urinary soluble VCAM-1 in systemic lupus erythematosus: a clinical marker for monitoring disease activity and damage. Clinical and Experimental Rheumatology, 2002, 20(3):403-406.

27. Sabry AA et al. Markers of inflammation and atherosclerosis in Egyptian patients with systemic lupus erythematosus. Nephrology, 2006, 11(4):329-335.

28. Frijns $\mathrm{R}$ et al. Persistent increase in plasma thrombomodulin in patients with a history of lupus nephritis: endothelial cell activation markers. Journal of Rheumatology, 2001, 28(3):514-519.

29. Ho CY et al. Elevated plasma concentrations of nitric oxide, soluble thrombomodulin and soluble vascular cell adhesion molecule-1 in patients with systemic lupus erythematosus. Rheumatology (Oxford), 2003, 42(1):117-122.

30. Mason JC, Kapahi P, Haskard DO. Detection of increased levels of circulating intercellular adhesion molecule 1 in some patients with rheumatoid arthritis but not in patients with systemic lupus erythematosus. Lack of correlation with levels of circulating vascular cell adhesion molecule 1. Arthritis and Rheumatism, 1993, 36(4):519-527. 